\title{
Propuesta de sistema de control basado en método ABC para determinar el stock de mercaderías en kalito distribuciones, Jaén 2021
}

Coronel Montoya Saly Yamir scoronelmo@ucvvirtual.edu.pe https://orcid.org/0000-0001-9039-5904

Gavidia Mondragón Lorenza lgavidiamo@ucvvirtual.edu.pe https://orcid.org/0000-0002-0233-6339

Mg. Roberto Carlos Oblitas Otero roblitas@ucvvirtual.edu.pe https://orcid.org/0000-0002-6541-7005

Universidad César Vallejo Moyobamba - Perú

\section{RESUMEN}

OBJETIVO: Determinar si este sistema de control permitirá determinar adecuadamente el stock de mercaderías en la empresa. METODOLOGÍA: Fue de tipo aplicada, de nivel correlacional, la técnica empleada fue el análisis documental y entrevista procesados mediante un programa estadístico. RESULTADOS: Los 3 objetivos específicos si se relacionan, el cual se observa que los procesos relacionados con el control de inventario no están estandarizados, y los responsables del almacén y de los procesos logísticos se basan únicamente en su experiencia. CONCLUSIÓN: Va a permitir llevar a cabo la correcta clasificación y distribución de las mercaderías, administrando de manera racional sus inventarios, e identificando aquellos productos que tienen una mayor demanda.

Palabras clave: sistema de control; método ABC de gestión de inventarios; stock de mercaderías. 


\title{
Proposal for a control system based on the $\mathrm{ABC}$ method to determine the stock of merchandise in kalito distribuciones, Jaén 2021
}

\begin{abstract}
OBJECTIVE: To determine if this control system will allow to adequately determine the stock of goods in the company. METHODOLOGY: It was applied type, correlational level, the technique used was the documentary analysis and interview processed through a statistical program. RESULTS: The 3 specific objectives are related, which is observed that the processes related to inventory control are not standardized, and those responsible for the warehouse and logistics processes are based solely on their experience. CONCLUSION: It will allow the correct classification and distribution of goods to be carried out, rationally managing their inventories, and identifying those products that have a greater demand.
\end{abstract}

Keywords: control system; $\mathrm{ABC}$ method of inventory management; stock of merchandise.

Artículo recibido: 05 octubre. 2021 Aceptado para publicación: 02 noviembre 2021

Correspondencia: salycmjm@gmail.com Conflictos de Interés: Ninguna que declarar 


\section{INTRODUCCIÓN}

Cabe destacar que una de las mayores debilidades que más han caracterizado a las empresas, es poder determinar con exactitud el control de sus inventarios, ya que en más de una ocasión estos se realizaron sin los correspondientes criterios técnicos, al no tenerse implementado una correcta herramienta de administración de almacén que logre llevar de manera adecuada el control de sus inventarios, y como es natural, ha conllevado a que estos negocios no puedan determinar con exactitud el stock de mercaderías.

Problemática local, se debe señalar que desde el inicio de sus actividades, la empresa "Kalito Distribuciones S.R.L." siempre ha tenido una serie de inconvenientes para implementar adecuadamente su sistema de gestión de inventarios, y que no le está permitiendo llevar de manera correcta el control de sus stocks de mercaderías, con los siguientes efectos adversos en el costo de sus servicios, que en más de una ocasión le ha venido afectando la determinación de sus precios, y observándose que el control de inventarios se hace de acuerdo al criterio de su propietario, sin los mínimos estudios técnicos que permitan establecer, si se están realizando de manera eficiente.

Tales negocios en su mayoría tienen que realizar de manera permanente, inventarios entre sus mercaderías adquiridas, recepcionadas, y comercializadas, a fin de establecer faltantes, mermas y desmedros, tanto por los generados en su almacenamiento, como en sus despachos. De la misma manera se puede mencionar que la problemática detectada en la Empresa Kalito Distribuciones, se circunscribe básicamente a las deficiencias en sus procesos logísticos y operativos, así como a la ineficiente infraestructura de su almacén. El trabajo de investigación se ha enfocado en la importancia de determinar la pertinencia de proponer un Sistema de control basado en el método ABC de gestión de inventarios, para determinar el stock de mercaderías en Kalito Distribuciones S.R.L., y que le permita llevar de manera eficaz el control de sus inventarios, y una vez obtenidos los resultados, ya estará en condiciones de demostrar las variables que más tendrán efecto en sus operaciones.

En tal sentido, la propuesta surge por la necesidad de estructurar un sistema de control basado en el método $\mathrm{ABC}$ de gestión de inventarios para determinar el stock de mercaderías en la "Empresa Kalito Distribuciones S.R.L." en la ciudad de Jaén en el periodo 2021, que vaya acorde con los resultados que se esperan obtener. Cabe destacar que la empresa inició sus actividades en el año 2021 como una Sociedad Comercial de 
Responsabilidad Limitada, cuyo giro principal es la venta al por mayor de productos electrodomésticos y otros enseres para el hogar. El domicilio fiscal del negocio es en Pasaje Teniente Pinglo N ${ }^{\circ} 151$ Sector Pueblo Nuevo (Mercado 28 de Julio). Se encuentra inscrita con código CIIU N ${ }^{\circ} 51414$ y se encuentra empadronada en el Registro Nacional de Proveedores para hacer contrataciones con el Estado Peruano.

\section{Problema General}

- ¿La Propuesta del Sistema de control basado en el método ABC para determinar el stock de mercaderías, le permitirá a la empresa Kalito Distribuciones S.R.L., determinar adecuadamente el stock de sus mercaderías, Jaén 2021 ?

\section{Problemas Específicos}

- ¿El Sistema de control basado en el método ABC de gestión de inventarios, mejorará el stock de mercaderías en la empresa Kalito Distribuciones S.R.L., Jaén en el año $2021 ?$

- ¿En qué medida el Sistema de control basado en el método ABC de gestión de inventarios, optimizará la gestión del almacén en la empresa Kalito Distribuciones S.R.L., Jaén $2021 ?$

- ¿La Propuesta del Sistema de control basado en el método ABC de gestión de inventarios, tendrá incidencia en la mejora de la información financiera en la empresa Kalito Distribuciones S.R.L., Jaén 2021 ?

\section{Objetivo General}

- Determinar si el Sistema de control basado en el método ABC de gestión de inventarios, permitirá determinar adecuadamente el stock de mercaderías en la empresa Kalito Distribuciones S.R.L., Jaén 2021.

\section{Objetivos Específicos}

- Identificar si el Sistema de control basado en el método ABC de gestión de inventarios, mejorará el stock de mercaderías en la empresa Kalito Distribuciones S.R.L., Jaén 2021.

- Establecer en qué medida el Sistema de control basado en el método ABC de gestión de inventarios, optimizará la gestión del almacén en la empresa Kalito Distribuciones S.R.L., Jaén 2021. 
- Analizar si la Propuesta del Sistema de control basado en el método ABC de gestión de inventarios, tendrá incidencia en la mejora de la información financiera en la empresa Kalito Distribuciones S.R.L., Jaén 2021.

\section{Hipótesis General}

- El Sistema de control basado en el método ABC de gestión de inventarios, permitirá determinar adecuadamente el stock de mercaderías en la empresa Kalito Distribuciones S.R.L., Jaén 2021.

\section{Hipótesis Específicas}

- El Sistema de control basado en el método ABC de gestión de inventarios, mejorará el stock de mercaderías en la empresa Kalito Distribuciones S.R.L, Jaén 2021.

- El Sistema de control basado en el método ABC de gestión de inventarios, optimizará la gestión del almacén en la empresa Kalito Distribuciones S.R.L., Jaén 2021

- La Propuesta del Sistema de control basado en el método ABC de gestión de inventarios, tendrá incidencia en la mejora de la información financiera en la empresa Kalito Distribuciones S.R.L., Jaén 2021.

\section{Antecedentes}

(Alvarado, 2018). En su tesis "El método ABC en el control de inventarios y su efecto en la rentabilidad de una microempresa que distribuye insumos de manufactura", en Ecuador, en la Universidad Técnica del Norte. La recomendación de la investigación es implementar el método de control de inventarios de clasificación ABC, en el cual se realiza el diagnóstico de situación técnica, de manera que se pueda determinar la situación actual de las microempresas. Esta investigación parte de un marco teórico en el que se conceptualiza la gestión de inventarios y el control que se debe dar.

Concluyó que, a través del manejo de la tecnología de recolección y proceso de datos, es posible determinar dónde se ubica el control de inventarios microempresariales, y los resultados obtenidos permiten la ejecución de los cálculos correspondientes para establecer los costos en donde incurrirá al mantener el control de inventarios a través de inventarios. Método de clasificación ABC.

En palabras de (Muñoz, 2017). En su tesis "Sistema de gestión de inventarios de platería La Isla E.U.”, en Colombia, en la Universidad Libre. Luego de analizar y diagnosticar la empresa, se constata que no existe tal sistema de gestión de inventarios en la empresa. Es por ello que no hay control o información insuficiente para comprar y reponer 
adecuadamente la gestión de productos agotados. Por falta de productos y baja demanda El inventario lento conduce a la pérdida de ventas, lo que conduce a grandes inversiones en productos almacenados.

Concluyó que, en base al método de clasificación de inventarios $\mathrm{ABC}$ se determinaron las referencias con mayor movimiento, estas referencias representaron solo el $20 \%$ del inventario y generaron el $80 \%$ de las ventas, y se estableció la forma más adecuada de administrarlas.

\section{Teorías}

Sistema de Control basado en Método ABC, según (Macías, León, \& Limón, 2019) es un método que pretende asignar de manera correcta los costos indirectos para mejorar los procesos, aumentando la utilidad de la empresa, entre otros. El método ABC clasifica y examina los artículos más significativos en el inventario de la empresa, discriminándolos de acuerdo a su demanda o a su precio unitario por consumo, es decir, estudiando su porcentaje mayor en el inventario total. (pág.86)

En el método $\mathrm{ABC}$ se constituyen tres categorías que especifican los productos según sus preferencias, estableciéndose los Artículos A (mayor importancia), los B (importancia secundaria) y C (poca importancia). No obstante, lo más importante de la clasificación es la identidad de los artículos de mayor condición y los artículos de escasa condición en los extremos de las categorías, por lo cual, el número de clases es inestable, así como el porcentaje de artículos en cada una de ellas. (Macías, León, \& Limón, 2019)

Los autores (Veloz Navarrete \& Parada Gutiérrez, 2017), citan a Ballou (2004), quien afirma que: La logística está vinculada a la gestión del flujo de material e información a lo largo del proceso de producción para mejorar la eficiencia y la satisfacción del cliente; por ello, mencionó los factores distintivos de las empresas del siglo XXI. En este caso, la única ventaja competitiva que se puede mantener durante mucho tiempo. Integrarlo en una cadena de suministro puede lograr este objetivo, que es más eficaz en general que otras cadenas de suministro que compiten en el mismo mercado.

(Sierra y Acosta, Guzmán Ibarra, \& García Mora, 2015) Sustentan que la gestión del almacén y el control de inventarios son habilidades y funciones que todo director de empresa debe alcanzar y distinguir para mejorar las operaciones logísticas de la empresa. El trabajo enfatiza la importancia de cada actividad y cuáles son las principales diferencias. 


\section{ESTRATEGIAS METODOLÓGICAS O MATERIALES Y MÉTODOS}

\section{Tipo y Diseño de Investigación}

La investigación es del tipo aplicada porque genera un análisis sobre un sistema de control en el método $\mathrm{ABC}$ de gestión de inventarios para determinar su stock de mercadería.

Su alcance es descriptivo correlacional, porque pretende identificar y precisar el stock de mercadería a través de un método con el objetivo de llevar un control real de inventario. La investigación presenta un diseño no experimental, ya que solo se basó en recolección de información para responder una hipótesis.

\section{Población}

Según la empresa Kalito Distribuciones S.R.L., la población está constituida por sus siete trabajadores.

\section{Muestra}

La muestra estudiada en el presente trabajo comprendió al Administrador y al Encargado del Almacén.

\section{Técnica e Instrumento de Recolección de Datos}

El instrumento utilizado para llevar a cabo un adecuado trabajo de campo, fue la guía de entrevista, esta se elaboró y aplicó al Administrador y al Encargado del Almacén.

\section{Procedimientos}

- Se realizó una reunión con el Administrador de la empresa Kalito Distribuciones S.R.L., a quien se le presentó una carta solicitando autorización para realizar el estudio.

- Las entrevistas se realizaron al Administrador y al Encargado del Almacén individualmente, durante el horario de receso. El tiempo para la actividad fue de sesenta minutos.

- Se procederá a determinar los indicadores de gestión de inventarios a fin de establecer su rotación, su demanda por temporadas, de la misma manera se plante la aplicación del Método de Control de Inventarios ABC para identificar los productos con mayor demanda.

- Una vez terminado el trabajo de campo, se transfirió la información a un Excel, donde se ordenó y clasificó la información oportuna, con la finalidad de facilitar la lectura, también se realizó un análisis de los resultados, en donde se tuvo información total del estudio. 


\section{Método de Análisis de Datos}

Para analizar los datos estadísticos de la técnica ejecutada se ha utilizado el software Microsoft Excel donde se realizó una tabla para tabular los datos recolectados y Gráfico para mostrar la cantidad representativa obtenida.

\section{RESULTADOS Y DISCUSIÓN}

\section{RESULTADOS}

De acuerdo a la aplicación de la entrevista al Administrador y Encargado del Almacén de empresa Kalito Distribuciones S.R.L., de la ciudad de Jaén, se dio a conocer lo siguiente: DIMENSIÓN 1 - Identificar los datos exactos de inventarios. ¿La posición de la mercadería y falta de documentación generan un ineficiente control, y que incide de manera inadecuada en la gestión de los inventarios?

\begin{tabular}{|l|c|c|}
\hline \multicolumn{1}{|c|}{ Escala } & Frecuencia & Porcentaje \\
\hline $\mathrm{Si}$ & 1 & $50 \%$ \\
No & 1 & $50 \%$ \\
\hline Total & $\mathbf{2}$ & $\mathbf{1 0 0 \%}$ \\
\hline
\end{tabular}

Fuente: Elaboración propia

Interpretación: Como se puede observar en la tabla 2 ante la pregunta; la posición de la mercadería y falta de documentación generan un ineficiente control, y que incide de manera inadecuada en la gestión de los inventarios, un 50\% que representa al Encargado de almacén de la empresa indica que sí, ya que no se conoce con exactitud la posición de las mercaderías en todo momento y sobre todo la falta de documentación que refleje sus movimientos, por otro lado, el Administrador que representa el otro 50\% indica que no, en razón de que el resultado de un ineficiente control se debe básicamente a la falta de herramientas informáticas que permita controlar los movimientos rutinarios de mercaderías, y sobre todo conocer adecuadamente su posición real dentro del almacén.

\section{DIMENSIÓN 2 - Aplicar Método}

¿Estarían dispuestos a implementar el Sistema de control basado en el método ABC de gestión de inventarios?

\begin{tabular}{|l|c|c|}
\hline \multicolumn{1}{|c|}{ Escala } & Frecuencia & Porcentaje \\
\hline $\mathrm{Si}$ & 2 & $100 \%$ \\
$\mathrm{No}$ & 0 & $0 \%$ \\
\hline Total & $\mathbf{2}$ & $\mathbf{1 0 0 \%}$ \\
\hline
\end{tabular}

Fuente: Elaboración propia 
Interpretación: Como se puede observar en la tabla 7 ante la pregunta; está de acuerdo con implementar el método $\mathrm{ABC}$ de gestión de inventarios que se basa de manera implícita en la regla del 80/20, el 100\% representado por el Administrador y Encargado de almacén asegura que sí, ya que la regla del 80/20 se considera un principio que permite determinar de manera puntual el esfuerzo realizado en la generación de las ventas, y su efecto en la determinación de los resultados, en otras palabras, el 20\% de los productos que comercializa la empresa van a generar el $80 \%$ del movimiento de las mercaderías, en tanto que el $80 \%$ de las mercaderías, van a generar el $20 \%$ de estos movimientos.

\section{DIMENSIÓN 3 - Determinación de Existencias}

¿Se encuentra debidamente estructurado el almacén de la empresa?

\begin{tabular}{|l|c|c|}
\hline \multicolumn{1}{|c|}{ Escala } & Frecuencia & Porcentaje \\
\hline $\mathrm{Si}$ & 0 & $0 \%$ \\
$\mathrm{No}$ & 2 & $100 \%$ \\
\hline Total & $\mathbf{2}$ & $\mathbf{1 0 0 \%}$ \\
\hline
\end{tabular}

Fuente: Elaboración propia

Interpretación: Como se puede observar en la tabla 10 ante la pregunta; Se encuentra debidamente estructurado el almacén de la empresa, el $100 \%$ representado por el Administrador y Encargado de almacén asegura que no, básicamente se encuentra estructurado para acoger las mercaderías que llegan a la empresa, conjuntamente con su descarga, control, manipulación, almacenaje, pesaje y picking, pero en más de una oportunidad se han presentado inconvenientes en su operatividad, debido a la ineficiente infraestructura de almacén.

\section{DIMENSIÓN 4 - Valorización de Existencias}

¿Se toman las medidas necesarias a fin de prevenir productos caducados o deteriorados?

\begin{tabular}{|lc|c|c|}
\hline \multicolumn{2}{|c|}{ Escala } & Frecuencia & Porcentaje \\
\hline $\mathrm{Si}$ & & 0 & $0 \%$ \\
$\mathrm{No}$ & & 2 & $100 \%$ \\
\hline \multicolumn{2}{|c|}{ Total } & $\mathbf{2}$ & $\mathbf{1 0 0 \%}$ \\
\hline
\end{tabular}

Fuente: Elaboración propia

Interpretación: Como se puede apreciar en la tabla 14 ante la pregunta; se toman las medidas necesarias a fin de prevenir productos caducados o deteriorados, el $100 \%$ representado por el Administrador y Encargado de almacén sostiene que no se toman las 
medidas que se requieren a fin de prevenir que las mercaderías se deterioren o caduquen, demostrando que está ocasionando pérdidas y mermas, por lo que no se está en condiciones de poder vender los productos ya que se encuentran en mal estado o vencidos.

\section{Discusión de los Resultados}

En este capítulo, se presenta la discusión de dichos resultados encontrados tomando en cuenta a los objetivos planteados, con apoyo de los antecedentes y teorías describiendo los fenómenos encontrados en alineación con la problemática definida.

Objetivo específico 1: Identificar si el Sistema de control basado en el método ABC de gestión de inventarios, mejorará el stock de mercaderías en la empresa Kalito Distribuciones S.R.L., Jaén 2021.

De acuerdo a la entrevista realizada al Administrador y Encargado de almacén, frente a la pregunta $\mathrm{N}^{\circ} 02$ ¿Se aplica un adecuado control de inventarios en la empresa?, se observó que la empresa carece de un adecuado control de inventarios, debido a que emplea controles simples que no refleja en detalle el movimiento de las mercaderías, y que como es natural, genera desorden y un acentuado desbalance financiero, así mismo, a causa de estos controles, no se conoce a profundidad la demanda de mercaderías, es decir, no se conoce con exactitud qué productos se comercializan más, y cuales se venden menos.

Objetivo específico 2: Establecer en qué medida el Sistema de control basado en el método $\mathrm{ABC}$ de gestión de inventarios, optimizará la gestión del almacén en la empresa Kalito Distribuciones S.R.L., Jaén 2021.

De acuerdo a la pregunta $\mathrm{N}^{\circ} 09$ ¿Se encuentra debidamente estructurado el almacén de la empresa?, se obtuvo que básicamente se encuentra estructurado para acoger las mercaderías que llegan a la empresa, conjuntamente con su descarga, control, manipulación, almacenaje, pesaje y picking, pero en más de una oportunidad se han presentado inconvenientes en su operatividad, debido a la ineficiente infraestructura de almacén.

Objetivo específico 3: Analizar si la Propuesta del Sistema de control basado en el método $\mathrm{ABC}$ de gestión de inventarios, tendrá incidencia en la mejora de la información financiera en la empresa Kalito Distribuciones S.R.L., Jaén 2021.

Realizando el análisis documental, se obtuvo información que las pérdidas generadas en los tres últimos años fueron por el inadecuado control y gestión de inventarios, 
ascendiendo aproximadamente al $6.3 \%$ del total de las mercaderías comercializadas, y a pesar de estos faltantes totalmente documentados, no se han tomado las medidas correctivas necesarias para evitar que estos faltantes se sigan sucediendo.

\section{CONCLUSIÓN O CONSIDERACIONES FINALES}

- Se llegó a determinar que el Sistema de control basado en el método ABC de gestión de inventarios, permite determinar adecuadamente el stock de mercaderías, así como sus actuales procesos de control y gestión en la empresa Kalito Distribuciones S.R.L., Jaén 2021.

- Se identificó que el Sistema de control basado en el método ABC de gestión de inventarios, mejorará el stock de mercaderías en la empresa Kalito Distribuciones S.R.L., a través de la aplicación de la propuesta la cual permitirá llevar a cabo el monitoreo periódico a cada producto comercializado, reduciendo por consiguiente las mermas que en más de una ocasión han sobrepasado el $2 \%$ de las mercaderías almacenadas.

- El Sistema de control basado en el método ABC de gestión de inventarios, optimizará la gestión del almacén en la empresa Kalito Distribuciones S.R.L., si bien se observa que el almacén en más de una oportunidad se ha tenido inconvenientes en su operatividad, por lo que el método $\mathrm{ABC}$ de gestión de inventarios va a permitir llevar a cabo la correcta clasificación y distribución de las mercaderías, administrando de manera racional sus inventarios, e identificando aquellos productos que tienen una mayor demanda.

- Se analizó que el método ABC de gestión de inventarios tendrá incidencia en la mejora de la información financiera, en razón de que permitirá conocer debidamente los procesos de adquisiciones, almacenamiento, abastecimiento y ventas, y sobre todo va a procurar que los inventarios sean administrados de manera eficaz y eficiente.

\section{Propuesta}

La propuesta permitirá desarrollar un conjunto de herramientas con la finalidad de resolver los inconvenientes que actualmente tiene la empresa en materia de control de sus inventarios, y en la inadecuada determinación del stock de sus mercaderías, procurando asimismo, resolver dichos problemas, mejorando por consiguiente el desempeño de sus procesos logísticos. 
El objetivo central de la propuesta es proporcionar a la empresa, los instrumentos necesarios para determinar de manera fehaciente el stock de sus mercaderías, así como llevar a cabo un eficaz control de su inventario.

En tal sentido, y con la finalidad de que se mantengan niveles óptimos del inventario de mercaderías, la empresa tendrá que cumplir necesariamente con los siguientes lineamientos:

- Implementar el Sistema de control basado en el método ABC de gestión de inventarios, que le permita determinar adecuadamente su stock de mercaderías.

- Llevar a cabo inventarios mensuales de sus inventarios, o en su defecto, realizar inventarios de manera inopinada, pero en tiempos bastante cortos.

- El inventario no deberá ser realizado por personal del almacén, sino por personal del área de contabilidad.

- Llevar un registro o reporte por cada inventario realizado, el mismo que contendrá toda la información requerida, como fecha del inventario, nombres, apellidos y firmas de los encargados del inventario, y en su caso, consignar debidamente los faltantes y sobrantes encontrados.

- De encontrarse diferencias entre la información reportada por parte del almacén, y el inventario consignado por el área de contabilidad, estas inconsistencias deberán informarse oportunamente a la administración para que tome los correctivos necesarios.

\section{Clasificación de los productos}

La empresa Kalito Distribuciones S.R.L. ofrece más de 1,200 productos y para describir cada uno de ellos, hemos recurrido al ejemplo de Clasificación de las Mercancías que hace la SUNAT - Aduana Peruana, para poder englobar todos los productos en los siguientes grupos:

- Por materia constitutiva.

- Por uso y función.

\section{Clasificación de los Productos - Materia Constitutiva}

\begin{tabular}{|l|l|c|}
\hline Serie & \multicolumn{1}{|c|}{ Denominación } & Aporte porcentual \\
\hline 001 & Productos plástico & \\
\hline 002 & Productos loza & $64 \%$ \\
\hline 003 & Productos de aluminio & \\
\hline 004 & Productos de acero & $23 \%$ \\
\hline 005 & Productos de vidrio & \\
\hline
\end{tabular}


Clasificación de los Productos - división el uso y función

\begin{tabular}{|c|c|c|}
\hline Serie & Denominación & Aporte porcentual \\
\hline 006 & Artefactos: cocina a gas & $13 \%$ \\
\hline
\end{tabular}

Distribución ABC de Familia de Artículos

\begin{tabular}{|l|l|c|c|c|}
\hline \multicolumn{1}{|c|}{ Productos } & $\begin{array}{c}\text { \% Del } \\
\text { Total }\end{array}$ & \% Acumulado & $\begin{array}{c}\text { Aporte } \\
\%\end{array}$ & $\begin{array}{c}\text { Clasificación } \\
\text { ABC }\end{array}$ \\
\hline Productos de plástico & $37 \%$ & $37 \%$ & & A \\
\hline Productos de loza & $14 \%$ & $51 \%$ & $64 \%$ & A \\
\hline Productos de aluminio & $13 \%$ & $64 \%$ & & A \\
\hline Productos de acero & $12 \%$ & $76 \%$ & $23 \%$ & B \\
\hline Productos de vidrio & $11 \%$ & $87 \%$ & & B \\
\hline Electrodomésticos & $7 \%$ & $94 \%$ & $13 \%$ & C \\
\hline artefactos: cocinas & $6 \%$ & $100 \%$ & & C \\
\hline
\end{tabular}

- En la clasificación “A”, se consignarán los productos que corresponden al grupo de familia de plásticos, loza y aluminio.

- En la clasificación "B", se consignarán los productos que corresponden al grupo de familia de acero y vidrios.

- En la clasificación "C", se consignarán los productos que corresponden al grupo de familia de televisores, refrigeradora, lavadora y cocinas a gas.

Una vez elaborada la clasificación " $A B C$ ”, se tendrá que hacer la redistribución de las mercaderías dentro del almacén:

- Los productos catalogados como "A" deberán estar ubicados cerca de la puerta de ingreso.

- Los productos catalogados como "B" deberán estar ubicados en puntos estratégicos.

- En tanto los productos catalogados como "C" deberán estar ubicados de manera indistinta.

\section{LISTA DE REFERENCIAS}

Alvarado,J. (2018). "The ABC method in inventory control and its effect on the profitability of a microenterprise distributor of inputs for manufacturing", in Ecuador, at the Universidad Técnica del Norte. Retrieved from: http://repositorio.utn.edu.ec/handle/123456789/8167 
Actualidad Empresa (2017). "Models and Strategies for inventory management and replenishments". Retrieved from:https://actualidadempresa.com/modelos-yestrategias-para-la-gestion-de-inventarios-y-aprovisionamientos/

Abanto. L. (2017), "Design of the distribution of the warehouse through $A B C$ methodology improving the reliability of the inventory information in the company tecni fluidos S.A.C.", published at the Señor de Sipán University. Retrieved from:http://repositorio.uss.edu.pe/bitstream/handle/uss/5126/Abanto\%20Zarate $\% 20$ Carlos\%20Eduardo.pdf?sequence=1\&isAllowed=y

Albujar \& Zapata (2014). "Design of an inventory system to reduce losses in the company TAILOY S.A.C.", published at the Señor de Sipán University. Retrieved from:http://repositorio.uss.edu.pe/bitstream/handle/uss/2294/ALBUJAR\%20AG UILAR\%20y\%20ZAPATA\%20MOYA.pdf?sequence $=1$

Arciniegas (2013), "Inventory management model for commercial companies in the city of Ibarra, province of Imbabura". Retrieved from:https://www.redalyc.org/pdf/5217/521752181003.pdf

Arreguin (2019). In Mexico "ABC Method of Inventory Control". Retrieved from:https://www.asnews.mx/noticias/metodo-abc-de-control-de-inventarios.

Allen, S. (2017). Stock management. Obtained from https://www.mheducation.es/bcv/guide/capitulo/8448199316.pdf

Alvarado, J. (2018). The ABC method in inventory control and its effect on the profitability of a microenterprise distributor of manufacturing inputs. Universidad Técnica del Norte, Ecuador. Obtenido de http://repositorio.utn.edu.ec/handle/123456789/8167

Andrada, B. (2017). Inventory control and its impact on the Financial Statements of the commercial company $M \& Q$ E.I.R.L. Universidad Peruana de las Américas, Lima. Obtained from http://repositorio.ulasamericas.edu.pe/bitstream/handle/upa/114/TRABAJO\%20 DE\%20INVESTIGACI\%c3\%93N\%20COMERCIAL\%20M\%26Q\%20EIRL.pdf ?sequence $=1 \&$ is Allowed $=\mathrm{y}$

Arellano, O., \& Quispe, G. (2017). Study of the Application of the ABC Cost Method in the MSEs of Ecuador. Scielo Peru. Obtenido de 
http://www.scielo.org.pe/scielo.php?script=sci_arttext\&pid=S231329572017000100004

Arias, E. (Diciembre de 2020). Applied research. Economipedia. Obtenido de https://economipedia.com/definiciones/investigacion-aplicada.html

Bravo, R., \& García, T. (2013). Control of the replenishment process for Stock Management and its impact on the level of customer service in a company. Redalyc. Obtained from https://www.redalyc.org/pdf/816/81632390004.pdf

Cárdenas Rabanal, K., Santisteban Atoche, S., Torres Goicochea, O., \& Pacheco Asmad, K. (2010). Efectos del control interno de inventarios en la rentabilidad de la empresa de reparación y fabricación metálica "Inversiones y servicios generales Jorluc S.A.C". Trujillo: Universidad Privada del Norte. Obtenido de https://upncontabilidad.files.wordpress.com/2011/01/efectos-del-control-internode-inventarios-en-la-rentabilidad-de-la-empresa-de-reparacic3b3n-yfabricaciones-metc3a1licas-inversiones-y-servicios-generales-jorluc-s-a-c.pdf

Casado, A. (09 de Enero de 2013). La Logística en Europa. Obtenido de https://mastrabajo.wordpress.com/2013/01/09/la-logistica-en-europa/

Chia Yep, A. (2018). Mejora de la gestión de inventarios del almacén de repuestos en Productos Tissue del Perú S.A. Lima: Universidad de San Martín de Porres. Obtenido de http://repositorio.usmp.edu.pe/handle/20.500.12727/4470

Consejo Nacional de Ciencia, Tecnología e Innovación Tecnológica. (2018). Reglamento de calificación, clasificación y registro de los Investigadores del sistema nacional de ciencia, tecnología e innovación tecnológica - Reglamento RENACYT. Lima, Perú: CONCYTEC. Obtenido de https://portal.concytec.gob.pe/images/renacyt/reglamento_renacyt_version_final. pdf

De Jesús Eugenio Barrionuevo, R. (2010). Logística de Inventario y su incidencia en las ventas de la Farmacia Cruz Azul "Internacional" de la ciudad de Ambato. Ambato: Universidad Técnica de Ambato. Obtenido de https://repositorio.uta.edu.ec/bitstream/123456789/1381/1/292\%20Ing.pdf

De La Cruz Rojas, R. A., \& Albujar Arango, M. J. (2014). Estrategias de control de inventarios para optimizar la producción y rentabilidad de la empresa Agro 
Macathon S.A.C. Lima: Universidad Autónoma del Perú. Obtenido de http://repositorio.autonoma.edu.pe/handle/AUTONOMA/151

Díaz Carvajal, D., \& Patiño Martínez, V. (2011). Implementación de sistema de gestión de inventarios para formas y color en láminas WJ ltda. Bogotá: Universidad Libre.

Obtenido de https://repository.unilibre.edu.co/bitstream/handle/10901/9188/PROYECTO\%20 -FINAL.pdf?sequence $=1$

Durán, Y. (2012). Administración del inventario. Redalyc, 58. Obtenido de https://www.redalyc.org/pdf/4655/465545892008.pdf

eserp. (s.f.). Los métodos de control de inventarios más usados. Obtenido de https://es.eserp.com/articulos/metodo-control-inventarios/

Garrido Bayas, I., \& Cejas Martínez, M. (2017). La gestión de inventario como factor estratégico en la administración de empresas. Obtenido de Revista Científica de Ciencias Gerenciales: https://dialnet.unirioja.es/servlet/articulo?codigo=7169805

Granda León, G., \& Rodríguez Gaybor, R. (2013). Diseño de un sistema de control basado en el Método ABC de gestión de inventarios, a través de indicadores de medición, aplicando a un estudio fotográfico en la ciudad de Machala. Guayaquil: Escuela Superior Politécnica Del Litoral. Obtenido de https://www.dspace.espol.edu.ec/xmlui/bitstream/handle/123456789/25082/TES IS\%20DE\%20GRADO\%20GRANDARODR\%c3\%8dGUEZ.pdf?sequence=2\&isAllowed $=\mathrm{y}$

Hemeryth Charpentier, F., \& Sánchez Gutiérrez, J. (2013). Implementación de un sistema de control interno operativo en los almacenes, para mejorar la gestión de inventarios de la constructora A\&A S.A.C. de la ciudad de Trujillo - 2013. Trujillo: Universidad Privada Antenor Orrego. Obtenido de http://repositorio.upao.edu.pe/bitstream/upaorep/140/1/HEMERYTH_FLAVIA_ IMPLEMENTACION_SISTEMA_CONTROL.pdf

Llayqui Saavedra, P. (2019). Propuesta e implementación de mejora de la gestión de inventarios para la optimización del área de almacén en la empresa UFITEC SAC en el periodo 2016-2017. Lima: Universidad de San Martín de Porres. Obtenido de http://repositorio.usmp.edu.pe/handle/20.500.12727/5445 
Logistics Cloud Applications. (s.f.). Control de Inventarios. Obtenido de Sistema de Inventario: https://www.linbis.com/es/sistema-de-control-de-inventarios/

Macías, R., León, A., \& Limón, C. (2019). Análisis de la cadena de suministro por clasificación ABC. Dialnet. Obtenido de file:///C:/Users/User/Downloads/Dialnet-

AnalisisDeLaCadenaDeSuministroPorClasificacionABC-6750256.pdf

Misari Argandoña, M. (2012). El control interno de inventarios y la gestión en las empresas de fabricación de calzado en el distrito de Santa Anita. LIma: Universidad de San Martín de Porres. Obtenido de http://repositorio.usmp.edu.pe/handle/20.500.12727/570 